\title{
Activity Coefficients at Infinite Dilution of Organic Compounds in Four New Imidazolium-Based Ionic Liquids
}

\author{
Jean-Charles Moïse, ${ }^{\dagger}$ Fabrice Mutelet, ${ }^{*,+}$ Jean-Noël Jaubert, ${ }^{\dagger}$ Laura M. Grubbs, ${ }^{\ddagger}$ William E. Acree, Jr., ${ }^{\neq}$and \\ Gary A. Baker ${ }^{\S}$ \\ ${ }^{\dagger}$ Laboratoire Reactions et Génie des Procédés, Nancy-Université, 1 rue Grandville, BP 2045154001 Nancy, France \\ ${ }^{\ddagger}$ Department of Chemistry, 1155 Union Circle \#305070, University of North Texas, Denton, Texas 76203-5017, United States \\ ${ }^{\S}$ Department of Chemistry, University of Missouri-Columbia, Columbia, Missouri 65211, United States
}

\begin{abstract}
Activity coefficients of 42 to 54 organic compounds in the four ionic liquids (ILs) 1-hexyl-3-methylimidazolium tris(pentafluoroethyl)trifluorophosphate, 1-butyl-3-methylimidazolium bis(pentafluoroethylsulfonyl)imide, 1,3-didecyl-2-methylimidazolium bis(trifluoromethylsulfonyl)imide, and 1-ethyl-3-methylimidazolium methanesulfonate were measured using inverse gas chromatography from ( 312 to 353 ) $\mathrm{K}$. The retention data were also converted to gas-to-IL and water-to-IL partition coefficients using the corresponding gas-to-water partition coefficients. Both sets of partition coefficients were analyzed using the modified Abraham solvation parameter model. The derived equations correlated the experimental gas-to-IL and water-to-IL partition coefficient data to within average standard deviations of 0.113 and 0.143 log units, respectively.
\end{abstract}

\section{INTRODUCTION}

New strategies are necessary for clean and efficient separation, purification, and enrichment processes in the biotechnology and (petro)chemical industries. For example, environmental restrictions regarding the quality of transportation fuels produced and the emissions from the refinery itself are currently foremost issues. Moreover, it has become clear that the burden imposed on present and future generations by fossil fuels is so heavy that the need to phase them out has become de facto. This imperative to reduce environmental pollution has ushered in a new generation of scientists focused on more eco-responsible methods. Among the new advances, ionic liquids (ILs) are being widely promoted as probable substitutes for traditional industrial solvents such as volatile organic compounds in a host of processes. Overall, as a class of solvent, ILs frequently combine the attractive features of excellent chemical stability, high thermal stability, and exceedingly low vapor pressure in a single solvent. ILs are commonly comprised of an asymmetric, bulky organic cation paired with a weakly coordinating anion that may be organic or inorganic in nature. The properties of the anion and functionality presented at the cationic or anionic site offer a means to alter the specific attributes of the solvent proper, giving rise to the notion of tuning, often in stepwise fashion, the key solvent features for the task at hand. Nowadays, it is widely accepted that ILs are among the most intriguing and diverse alternative media available not only for conventional solvent-driven chemical processes like synthesis and (bio)catalysis, but also next-generation electrolytes, lubricants, and modifiers of mobile and stationary phases within the separation sciences. ${ }^{1-3}$ Numerous works have shown that a large number of ILs exhibit selectivities and capacities better than the solvents typically employed to solve industrial separation problems. ${ }^{4-10}$ For instance, IL-assisted extractive distillation or liquid-liquid extraction forms a powerful approach in the separation of ethanol-water mixtures ${ }^{11}$ and thiophene from aliphatic hydrocarbons. ${ }^{12}$
The thermodynamic properties of dialkylimidazolium-based ILs are relatively well-described in the literature. ${ }^{13-20}$ Nevertheless, there is a paucity of data concerning functional or taskspecific ILs. Moreover, the sheer number of individual ILs (and classes thereof) that attract attention has grown considerably in the last couple of years.

Up to now, few experimental separation data exist for tris(pentafluoroethyl)trifluorophosphate ILs, despite the fact that ILs containing the tris (perfluoroalkyl)trifluorophosphate (FAP) anion show excellent hydrolytic, thermal, and electrochemical stability and provide numerous advantages in organic synthesis, electrosynthesis, gas sorption, and battery applications. ${ }^{21-26}$ One of the most peculiar properties exhibited by these ILs is their prominent hydrophobic nature. ${ }^{27}$ Compared to other commonly used ILs for extractions, such as those containing the hexafluorophosphate $\left(\left[\mathrm{PF}_{6}\right]^{-}\right)$or bis(trifluoromethylsulfonyl)imide $\left(\left[\mathrm{Tf}_{2} \mathrm{~N}\right]^{-}\right)$anion, FAP-based ILs are substantially more hydrophobic and hydrolytically stable, ${ }^{28}$ making them ideal candidates for the study of direct immersion extraction from aqueous matrixes.

This study is a continuation of our investigations into the thermodynamic properties of dialkylimidazolium and functionalized ILs. $^{4-10,29}$ In previous work, we have shown that the introduction of polar chains into an IL strongly affects its behavior and interaction with organic compounds. For example, appending short polar chains to an imidazolium cation of an IL yields increased selectivity toward mixtures containing $\{$ alcohol + aliphatic $\}$ or $\{$ aromatic + aliphatic $\}$ solutes. This work is focused on the behavior of four new imidazolium-based ILs: 1-hexyl-3-methylimidazolium tris(pentafluoroethyl)trifluorophosphate, $\left([\mathrm{HMIm}]^{+}[\mathrm{FAP}]^{-}\right), 1$-butyl-3-methylimidazolium

\section{Received: February 23, 2011}

Accepted: $\quad$ May 18, 2011

Published: June 02, 2011 

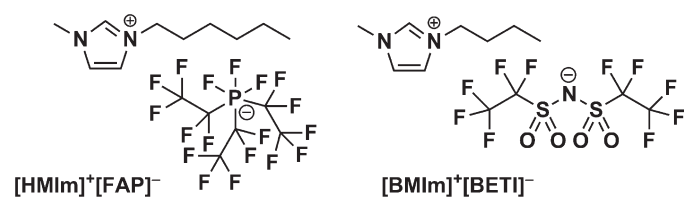

$[\mathrm{BMIm}]^{+}[\mathrm{BETI}]^{-}$

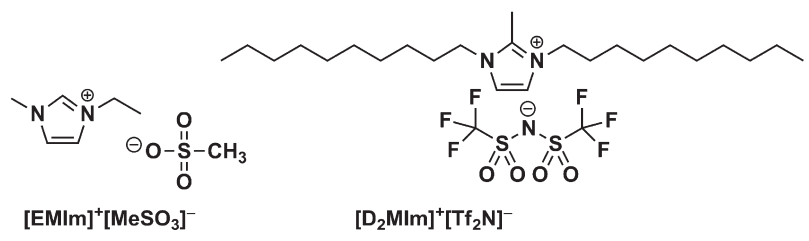

Figure 1. Chemical structures of the ILs investigated in this work.

bis(pentafluoroethylsulfonyl)imide, $\left([\mathrm{BMIm}]^{+}[\mathrm{BETI}]^{-}\right), 1,3-$ didecyl-2-methylimidazolium bis(trifluoromethylsulfonyl)imide, $\left(\left[\mathrm{D}_{2} \mathrm{MIm}\right]^{+}\left[\mathrm{Tf}_{2} \mathrm{~N}\right]^{-}\right)$, and 1-ethyl-3-methylimidazolium methanesulfonate $\left([\mathrm{EMIm}]^{+}\left[\mathrm{MeSO}_{3}\right]^{-}\right)$. The molecular structures of these ILs are given in Figure 1. In this work, gas-liquid chromatography was used to quantify intermolecular solute-IL interactions and to predict their potential in various extraction and extractive distillation processes.

Using linear solvation energy relationships (LSERs) and retention data, one can quantify various intermolecular solute- $\mathrm{IL}$ interactions. Along these lines, Acree and co-workers have reported mathematical correlations based on the general Abraham solvation parameter model for both the gas-to-solvent partition coefficient, $K_{\mathrm{L}}$, and the water-to-solvent partition coefficient, $P .^{30-32}$ Sprunger et al. ${ }^{33-35}$ later modified the Abraham solvation parameter model as

$$
\begin{aligned}
\log K_{\mathrm{L}}= & c_{\text {cation }}+c_{\text {anion }}+\left(e_{\text {cation }}+e_{\text {anion }}\right) \cdot E+\left(s_{\text {cation }}+s_{\text {anion }}\right) \cdot S \\
& +\left(a_{\text {cation }}+a_{\text {anion }}\right) \cdot A+\left(b_{\text {cation }}+b_{\text {anion }}\right) \cdot B+\left(l_{\text {cation }}+l_{\text {anion }}\right) \cdot L
\end{aligned}
$$

$$
\begin{aligned}
\log P= & c_{\text {cation }}+c_{\text {anion }}+\left(e_{\text {cation }}+e_{\text {anion }}\right) \cdot E+\left(s_{\text {cation }}+s_{\text {anion }}\right) \cdot S \\
& +\left(a_{\text {cation }}+a_{\text {anion }}\right) \cdot A+\left(b_{\text {cation }}+b_{\text {anion }}\right) \cdot B+\left(v_{\text {cation }}+v_{\text {anion }}\right) \cdot V
\end{aligned}
$$

recasting each of the six solvent equation coefficients as a summation of their respective cation and anion contributions. It is important to note that, by splitting the system constants into ion-specific contributions, these authors make the assumption that each solute-ion interaction is unaffected by the chemical nature of the counterion present in the IL. We justify this choice in the following section.

The dependent variables in eqs 1 and 2 are solute descriptors as follows: $E$ and $S$ refer to the excess molar refraction in units of $\left(\mathrm{cm}^{3} \cdot \mathrm{mol}^{-1}\right) / 10$ and a dipolarity/polarizability description of the solute, respectively, $A$ and $B$ are measures of the solute hydrogen-bond acidity and basicity, $V$ is the McGowan volume in units of $\left(\mathrm{cm}^{3} \cdot \mathrm{mol}^{-1}\right) / 100$, and $L$ is the logarithm of the gas-tohexadecane partition coefficient at $298 \mathrm{~K}$. Sprunger et al. calculated equation coefficients for 8 cations and 4 anions using a database that contained 584 experimental $\log K_{\mathrm{L}}$ and 571 experimental $\log P$ values. Importantly, no loss in predictive accuracy was observed by separating the equation coefficients into individual cation-specific and anion-specific values. Of course, the major advantage of splitting the equation coefficients into individual cation- and anion-specific contributions is that one can begin to make predictions for far more ILs based on permutations comprising these specific ions.

\section{EXPERIMENTAL PROCEDURES AND RESULTS}

Materials and Reagents. 1-Ethyl-3-methylimidazolium methanesulfonate $\left([\mathrm{EMIm}]^{+}\left[\mathrm{MeSO}_{3}\right]^{-}\right.$, Alfa Aesar Chemicals, 0.99 mass fraction) was dried at $353 \mathrm{~K}$ for several days at reduced pressure to remove volatile impurities and trace water, yielding a water content below 0.02 mass fraction, as determined by Karl Fisher titration. High purity 1-hexyl-3-methylimidazolium tris(pentafluoroethyl)trifluorophosphate $\left([\mathrm{HMIm}]^{+}[\mathrm{FAP}]^{-}\right)$ from EMD was similarly dried before use. Ultrapure, spectroscopy-grade 1-butyl-3-methylimidazolium bis(pentafluoroethylsulfonyl)imide $\left([\mathrm{BMIm}]^{+}[\mathrm{BETI}]^{-},>0.999\right.$ mass fraction) was synthesized and dried following established methods. ${ }^{36,37} 1,3-$ Didecyl-2-methylimidazolium bis(trifluoromethylsulfonyl)imide $\left(\left[\mathrm{D}_{2} \mathrm{MIm}\right]^{+}\left[\mathrm{Tf}_{2} \mathrm{~N}\right]^{-}\right)$was prepared from 1,3-didecyl-2methylimidazolium chloride $\left(\left[\mathrm{D}_{2} \mathrm{MIm}\right]^{+} \mathrm{Cl}^{-}, 0.96\right.$, Sigma $)$ and lithium bis(trifluoromethylsulfonyl)imide $\left(\mathrm{Li}^{+}\left[\mathrm{Tf}_{2} \mathrm{~N}\right]^{-}, 3 \mathrm{M}\right)$. In a typical preparation, $\left[\mathrm{D}_{2} \mathrm{MIm}\right]^{+} \mathrm{Cl}^{-}$was combined with 1.05 equivalents of $\mathrm{Li}^{+}\left[\mathrm{Tf}_{2} \mathrm{~N}\right]^{-}$in a $1: 1(\mathrm{v} / \mathrm{v})$ dichloromethanewater system to produce an initially milky-white upper phase and a hazy bottom layer. After vigorous shaking overnight, the biphase was allowed to reestablish. The now-clear aqueous phase was discarded, and the organic phase washed several additional times with doubly distilled deionized water, using a separatory funnel, until the washes tested negative for the presence of halide using concentrated silver nitrate reagent. After two additional washes with warm deionized water, the organic phase was dried using a rotary evaporator, followed by gentle further drying on a Schenk line overnight at $333 \mathrm{~K}$ to give $\left[\mathrm{D}_{2} \mathrm{MIm}\right]^{+}\left[\mathrm{Tf}_{2} \mathrm{~N}\right]^{-}$in $\sim 95 \%$ isolated yield.

In addition to the treatment mentioned above, each IL was further purified by subjecting the liquid to a very low pressure of about $5 \mathrm{~Pa}$ at $343 \mathrm{~K}$ for approximately $24 \mathrm{~h}$. Next, packed columns were conditioned for a $12 \mathrm{~h}$ duration. Based upon our experience, we can safely assume that this procedure adequately removes any volatile chemicals and moisture from the IL and Chromosorb. Beyond this soft thermal treatment, no other attempts were made to analyze or specifically identify the impurities remaining within the ILs. Test solutes were purchased from Aldrich at a purity $\geq 99.5 \%$ and were used without further purification because our gas-liquid chromatography technique efficiently separates any impurities on the column.

Apparatus and Experimental Procedure. Inverse chromatography experiments were carried out using a Varian CP-3800 gas chromatograph equipped with a heated on-column injector and a flame ionization detector. The injector and detector temperatures were kept at $523 \mathrm{~K}$ during all experiments. The helium flow rate was adjusted to obtain adequate retention times. Methane was used to determine the column hold-up time. Exit gas flow rates were measured with a soap bubble flow meter. The temperature of the oven was determined with a Pt100 probe and controlled to within $\pm 0.1 \mathrm{~K}$. A personal computer directly recorded detector signals, and the corresponding chromatograms were generated using Galaxie software.

Using a rotary evaporation preparatory technique, $1.0 \mathrm{~m}$ length columns were packed with a stationary phase consisting of 0.20 to 0.35 mass fraction of IL on Chromosorb WHP (60-80 mesh). After the evaporation of chloroform in vacuo, the support was equilibrated at $333 \mathrm{~K}$ during $6 \mathrm{~h}$. Before conducting 
measurements, each packed column was conditioned for $12 \mathrm{~h}$ at $363 \mathrm{~K}$ with a flow rate of $20 \mathrm{~cm}^{3} \cdot \mathrm{min}^{-1}$. The packing level was calculated from the masses of the packed and empty columns and was checked throughout experiments. The masses of the stationary phase were determined to a precision of $\pm 0.0003 \mathrm{~g}$. A (1 to 5) $\mu$ L volume of the headspace sample vapor was injected to satisfy infinite dilution conditions, and each experiment was repeated at least twice to confirm reproducibility. Retention times were generally rigorously reproducible to within (0.01 to $0.03) \mathrm{min}$. To verify stability under these experimental conditions, ruling out elution of the stationary phase by the helium stream, measurements of retention time were repeated systematically each day for three selected typical solutes. No changes in the retention times were observed during this study.

Theoretical Basis. The retention data garnered by inverse chromatography experiments were used to calculate partition coefficients of the numerous solutes in the different ILs. The net retention volume, $V_{\mathrm{N}}$, was calculated via the typical relationship as follows: ${ }^{38}$

$$
V_{\mathrm{N}}=\frac{3}{2} \cdot \frac{\left[\left(\frac{P_{i}}{P_{\mathrm{o}}}\right)^{2}-1\right]}{\left[\left(\frac{P_{i}}{P_{\mathrm{o}}}\right)^{3}-1\right]} \cdot U_{0} \cdot t_{\mathrm{R}}^{\prime} \cdot \frac{T_{\mathrm{col}}}{T_{\mathrm{r}}} \cdot\left(1-\frac{P_{\mathrm{ow}}}{P_{\mathrm{o}}}\right)
$$

The adjusted retention time, $t_{\mathrm{R}}{ }^{\prime}$, was taken as the difference between the retention time of a particular solute and that of methane, $T_{\text {col }}$ is the column temperature, $U_{0}$ is the flow rate of the carrier gas measured at room temperature $\left(T_{\mathrm{r}}\right), P_{\text {ow }}$ is the vapor pressure of water at $T_{\mathrm{r}}$, and $P_{\mathrm{i}}$ and $P_{\mathrm{o}}$ are the inlet and outlet pressures, respectively.

Activity coefficients at infinite dilution for solute 1 in IL $2, \gamma_{1,2}^{\infty}$, were calculated with the following expression: ${ }^{38}$

$$
\ln \gamma_{1,2}^{\infty}=\ln \left(\frac{n_{2} R T}{V_{\mathrm{N}} \cdot P_{1}^{0}}\right)-P_{1}^{0} \cdot \frac{B_{11}-V_{1}^{0}}{R T}+\frac{2 B_{13}-V_{1}^{\infty}}{R T} \cdot J \cdot P_{0}
$$

where $n_{2}$ is the number of moles of stationary phase component within the column, $R$ is the gas constant, $T$ is the oven temperature, $B_{11}$ is the second virial coefficient of the solute in the gaseous state at temperature $T, B_{13}$ is the mutual virial coefficient between solute 1 and the carrier gas (helium, denoted by " 3 "), and $P_{1}^{0}$ is the probe vapor pressure at temperature $T$. All thermodynamic properties of the pure solutes needed for these calculations were given in previous work. ${ }^{4}$

Gas-to-IL partition coefficients, $K_{\mathrm{L}}$, used in the LSER approach were calculated using the expression

$$
K_{\mathrm{L}}=\frac{R T}{\gamma_{1,2}^{\infty} P_{1}^{0} V_{\text {solvent }}}
$$

The corresponding water-to-IL partition coefficients, $P$, were calculated through eq 6

$$
\log P=\log K_{\mathrm{L}}-\log K_{\mathrm{w}}
$$

which requires knowledge of the solute's gas-phase partition coefficient into water, $K_{\mathrm{w}}$, which is available for most of the solutes studied here. Water-to-IL partition coefficients calculated through eq 6 pertain to a hypothetical partitioning process involving solute transfer from water to the anhydrous IL. For convenience, we have collected these partition coefficients in the last two columns of Tables 1 to 4 .

\section{RESULTS AND DISCUSSION}

Activity Coefficients at Infinite Dilution of Organic Compounds in Ionic Liquids. The errors in the experimental determination of activity coefficient are evaluated to be about $3 \%$. For all ILs studied in this work, no interfacial adsorption was observed while the average relative standard deviation between data sets obtained from different packed columns was about (3 to 4 ) \%. Experimental activity coefficients at infinite dilution calculated using eqs 3 to 5 are listed in Tables 1 to 4 . The ILs studied show similar behaviors to typical dialkylimidazolium ILs. Activity coefficients at infinite dilution for most organic compounds decrease with an increase in temperature. Of the series of organic compounds studied, alkanes exhibit the lowest solubility in these four ILs. The solubility of apolar compounds increases with the increase of the alkyl chain length grafted onto the imidazolium cation. For compounds containing the same number of carbon atoms but originating from different solute families, it was observed that $\gamma_{\text {alcohol }}<\gamma_{\text {aromatic }}<\gamma_{\text {alkyne }}<\gamma_{\text {alkene }}<\gamma_{\text {alkane. }}$. This overall trend is followed for all ILs, regardless of the cation or anion identity. It is noteworthy that the presence of multiple bonds within the solute increases solubility considerably.

In general, the infinite dilution activity coefficients of the alcohols are relatively small, with the solubility of alcohols and chloroalkanes being higher in $[\mathrm{EMIm}]^{+}\left[\mathrm{MeSO}_{3}\right]^{-}$than in the other ILs studied. The hydroxyl group can potentially interact with either the anion and/or the cation of the IL. Interestingly, these classes of compound follow similar trends as the hydrocarbons. Branched-chain alcohols have a lower solubility compared to linear alcohols, and their activity coefficients increase with increasing chain length. Notably, ketones and aldehydes strongly interact with ILs and thus show even higher solubilities.

In Table 5, activity coefficients measured at infinite dilution for selected organic solutes in $[\mathrm{EMIm}]^{+}\left[\mathrm{MeSO}_{3}\right]^{-}$from this work are compared with recent data published by Blahut et al. ${ }^{39}$ Reasonably good agreement is observed with a standard deviation of about $6 \%$ for polar compounds, although the deviation is closer to $30 \%$ for $n$-alkanes.

Selectivities and capacities at infinite dilution calculated from activity coefficients respectively, $S_{12}^{\infty}$ and $k_{1}^{\infty}$, are reported in Table 6 for illustrative separation problems at $323.15 \mathrm{~K}$ : hexane/ benzene, hexane/methanol, hexane/thiophene, and cyclohexane/thiophene:

$$
\begin{gathered}
S_{12}^{\infty}=\frac{\gamma_{1 / \mathrm{RTIL}}^{\infty}}{\gamma_{2 / \mathrm{RTIL}}^{\infty}} \\
k_{1}^{\infty}=\frac{1}{\gamma_{1 / \mathrm{RTIL}}^{\infty}}
\end{gathered}
$$

Concerning the separation of benzene from hexane, the selectivities obtained using $[\mathrm{HMIm}]^{+}[\mathrm{FAP}]^{-}$and $[\mathrm{EMIm}]^{+}\left[\mathrm{MeSO}_{3}\right]^{-}$are of the same order of magnitude as for classical solvents used in industry like sulfolane (30.5), dimethylsulfoxide (22.7), and $N$-methyl-2-pyrrolidinone (12.5). Nevertheless, the capacity of $[\mathrm{EMIm}]^{+}\left[\mathrm{MeSO}_{3}\right]^{-}$is lower than those observed with other ILs. A better capacity may be obtained by a moderate lengthening in the alkyl chain grafted to the 
Table 1. Infinite Dilution Activity Coefficients and Logarithm of Partition Coefficients, $\log K_{\mathrm{L}}$ and $\log P$, of Organic Compounds in $[\mathrm{HMIm}]^{+}[\mathrm{FAP}]$

\begin{tabular}{|c|c|c|c|c|c|}
\hline \multirow[b]{2}{*}{ solute } & \multicolumn{3}{|c|}{$\gamma^{\infty}$} & \multirow{2}{*}{\multicolumn{2}{|c|}{$\frac{\log K_{\mathrm{L}} \quad \log P}{298.15 \mathrm{~K}}$}} \\
\hline & $312.80 \mathrm{~K}$ & $332.85 \mathrm{~K}$ & $354.65 \mathrm{~K}$ & & \\
\hline hexane & 6.393 & 5.693 & 4.409 & 1.601 & 3.421 \\
\hline 3-methylpentane & 5.629 & 5.055 & 4.430 & 1.591 & 3.431 \\
\hline heptane & 8.844 & 7.733 & 6.624 & 2.000 & 3.960 \\
\hline 2,2,4-trimethylpentane & 7.982 & 7.130 & 6.180 & 2.019 & 4.139 \\
\hline octane & 12.207 & 10.534 & 8.867 & 2.365 & 4.475 \\
\hline nonane & 17.681 & 15.467 & 12.441 & 2.747 & 4.897 \\
\hline decane & 22.707 & 19.044 & 15.263 & 3.090 & 5.410 \\
\hline undecane & 30.977 & 25.474 & 19.895 & 3.455 & 5.835 \\
\hline dodecane & 39.253 & 33.077 & 25.673 & 3.826 & 6.356 \\
\hline methylcyclopentane & 4.580 & 4.086 & 3.522 & 1.816 & 2.986 \\
\hline cyclohexane & 4.782 & 4.166 & 3.615 & 1.942 & 2.842 \\
\hline methylcyclohexane & 5.886 & 5.231 & 4.494 & 2.176 & 3.386 \\
\hline cycloheptane & 9.005 & 13.241 & 28.671 & 2.482 & 3.072 \\
\hline benzene & 0.517 & 0.552 & 0.564 & 2.984 & 2.354 \\
\hline toluene & 0.674 & 0.727 & 0.753 & 3.386 & 2.736 \\
\hline ethylbenzene & 0.956 & 1.032 & 1.043 & 3.687 & 3.107 \\
\hline$m$-xylene & 0.939 & 0.997 & 1.010 & 3.773 & 3.163 \\
\hline$p$-xylene & 0.992 & 1.034 & 1.000 & 3.719 & 3.129 \\
\hline$o$-xylene & 0.888 & 0.947 & 0.957 & 3.891 & 3.151 \\
\hline 1-hexene & 3.668 & 3.422 & 3.142 & 1.801 & 2.961 \\
\hline 1-hexyne & 1.938 & 1.886 & 1.755 & 2.254 & 2.464 \\
\hline 1-heptyne & 2.545 & 2.501 & 2.318 & 2.625 & 3.065 \\
\hline 2-butanone & 0.204 & 0.195 & 0.171 & 3.402 & 0.682 \\
\hline 2-pentanone & 0.271 & 0.298 & 0.311 & 3.713 & 1.133 \\
\hline 3-pentanone & 0.236 & 0.280 & 0.301 & 3.739 & 1.239 \\
\hline 1,4-dioxane & 0.273 & 0.327 & 0.339 & 3.708 & -0.002 \\
\hline methanol & 1.571 & 2.006 & 1.581 & 2.333 & -1.407 \\
\hline ethanol & 2.095 & 1.688 & 1.312 & 2.482 & -1.188 \\
\hline 1-propanol & 2.467 & 1.958 & 1.466 & 2.842 & -0.718 \\
\hline 2-propanol & 2.061 & 1.622 & 1.259 & 2.614 & -0.866 \\
\hline 2-methyl-1-propanol & 2.772 & 2.134 & 1.621 & 3.050 & -0.250 \\
\hline 1-butanol & 4.903 & 2.324 & 1.756 & 2.984 & -0.476 \\
\hline trifluoroethanol & 0.987 & 0.891 & 0.728 & 2.755 & -0.405 \\
\hline diethyl ether & 0.837 & 0.866 & 0.897 & 2.029 & 0.739 \\
\hline diisopropyl ether & 1.591 & 1.696 & 1.719 & 2.298 & 1.248 \\
\hline chloroform & 1.092 & 1.090 & 1.054 & 2.324 & 1.534 \\
\hline dichloromethane & 0.660 & 0.699 & 0.707 & 2.068 & 1.108 \\
\hline tetrachloromethane & 2.191 & 2.078 & 1.939 & 2.243 & 2.430 \\
\hline acetonitrile & 0.295 & 0.295 & 0.292 & 3.246 & 0.396 \\
\hline nitromethane & 0.452 & 0.438 & 0.410 & 3.433 & 0.483 \\
\hline 1-nitropropane & 0.452 & 0.451 & 0.436 & 3.992 & 1.542 \\
\hline triethylamine & 2.093 & 1.955 & 1.758 & 2.490 & 0.130 \\
\hline pyridine & 0.302 & 0.326 & 0.331 & 3.873 & 0.433 \\
\hline thiophene & 0.577 & 0.602 & 0.604 & 3.005 & 1.965 \\
\hline formaldehyde & 0.096 & 0.134 & 0.146 & & \\
\hline propionaldehyde & 0.274 & 0.283 & 0.285 & 2.749 & 0.229 \\
\hline
\end{tabular}

imidazolium cation. Indeed, longer alkyl chains are observed to increase the capacity, but with detriment to selectivity, as seen with $\left[\mathrm{D}_{2} \mathrm{MIm}\right]^{+}\left[\mathrm{Tf}_{2} \mathrm{~N}\right]^{-} \cdot[\mathrm{EMIm}]^{+}\left[\mathrm{MeSO}_{3}\right]^{-}$behaves
Table 2. Infinite Dilution Activity Coefficients and Logarithm of Partition Coefficients, $\log K_{\mathrm{L}}$ and $\log P$, of Organic Compounds in $[\mathrm{BMIm}]^{+}[\mathrm{BETI}]$

\begin{tabular}{|c|c|c|c|c|c|}
\hline \multirow{3}{*}{ solute } & \multicolumn{3}{|c|}{$\gamma^{\infty}$} & \multirow{2}{*}{\multicolumn{2}{|c|}{$\frac{\log K_{\mathrm{L}} \log P}{298.15 \mathrm{~K}}$}} \\
\hline & $322.55 \mathrm{~K}$ & $342.75 \mathrm{~K}$ & $362.65 \mathrm{~K}$ & & \\
\hline & 9.490 & 8.798 & 8.430 & 1.341 & 3.161 \\
\hline 3-methylpentane & 8.232 & 7.793 & 7.616 & 1.320 & 3.160 \\
\hline heptane & 13.633 & 12.370 & 11.625 & 1.686 & 3.646 \\
\hline 2,2,4-trimethylpentane & 13.282 & 11.712 & 10.496 & 1.641 & 3.761 \\
\hline octane & 20.374 & 17.316 & 15.542 & 1.984 & 4.094 \\
\hline nonane & 31.509 & 25.997 & 21.805 & 2.317 & 4.467 \\
\hline decane & 37.382 & 33.298 & 27.989 & 2.715 & 5.035 \\
\hline undecane & 57.328 & 45.877 & 37.821 & 2.999 & 5.379 \\
\hline dodecane & 77.801 & 62.004 & 50.931 & 3.338 & 5.868 \\
\hline tridecane & 111.158 & 78.063 & 68.856 & 3.688 & \\
\hline tetradecane & 153.275 & 112.679 & 91.528 & 4.011 & \\
\hline methylcyclopentane & 6.847 & 6.055 & 5.792 & 1.513 & 2.683 \\
\hline cyclohexane & 7.002 & 6.121 & 5.405 & 1.614 & 2.514 \\
\hline methylcyclohexane & 9.090 & 7.960 & 7.065 & 1.827 & 3.077 \\
\hline cycloheptane & 19.825 & 35.188 & 56.459 & 2.114 & 2.694 \\
\hline benzene & 1.012 & 1.016 & 1.029 & 2.556 & 1.926 \\
\hline toluene & 1.397 & 1.417 & 1.456 & 2.931 & 2.281 \\
\hline ethylbenzene & 2.043 & 2.052 & 2.062 & 3.221 & 2.641 \\
\hline$m$-xylene & 2.024 & 2.026 & 2.090 & 3.302 & 2.692 \\
\hline$p$-xylene & 2.049 & 2.034 & 2.002 & 3.277 & 2.687 \\
\hline$o$-xylene & 1.873 & 1.890 & 1.965 & 3.434 & 2.774 \\
\hline 1-hexene & 6.065 & 5.546 & 5.242 & 1.437 & 2.597 \\
\hline 1-hexyne & 2.859 & 2.729 & 2.639 & 1.958 & 2.168 \\
\hline 1-heptyne & 3.914 & 3.761 & 3.576 & 2.306 & 2.746 \\
\hline 2-butanone & 0.453 & 0.389 & 0.351 & 2.905 & 0.185 \\
\hline 2-pentanone & 0.666 & 0.686 & 0.701 & 3.193 & 0.613 \\
\hline 3-pentanone & 0.624 & 0.656 & 0.695 & 3.182 & 0.682 \\
\hline 1,4-dioxane & 0.429 & 0.607 & 0.576 & 3.427 & -0.283 \\
\hline methanol & 1.234 & 0.997 & 0.942 & 2.263 & -1.477 \\
\hline ethanol & 1.647 & 1.367 & 1.179 & 2.437 & -1.233 \\
\hline 1-propanol & 1.991 & 1.622 & 1.349 & 2.786 & -0.774 \\
\hline 2-propanol & 1.766 & 1.456 & 1.249 & 2.526 & -0.954 \\
\hline 2-methyl-1-propanol & 2.264 & 1.838 & 1.574 & 2.978 & -0.322 \\
\hline 1-butanol & 2.493 & 1.997 & 1.704 & 3.167 & -0.293 \\
\hline trifluoroethanol & 0.457 & 0.412 & 0.373 & 2.962 & -0.198 \\
\hline diethyl ether & 1.715 & 1.704 & 1.653 & 1.564 & 0.394 \\
\hline diisopropyl ether & 3.462 & 3.458 & 3.427 & 1.817 & 1.817 \\
\hline chloroform & 0.613 & 0.740 & 0.790 & 2.530 & 1.740 \\
\hline dichloromethane & 0.356 & 0.436 & 0.539 & 2.308 & 1.348 \\
\hline tetrachloromethane & 2.403 & 2.349 & 2.330 & 2.087 & 2.277 \\
\hline acetonitrile & 0.528 & 0.514 & 0.504 & 2.855 & 0.005 \\
\hline nitromethane & 0.625 & 0.682 & 0.699 & 3.216 & 0.266 \\
\hline 1-nitropropane & 0.769 & 0.821 & 0.756 & 3.620 & 1.170 \\
\hline triethylamine & 2.735 & 3.614 & 3.268 & 2.322 & -0.038 \\
\hline pyridine & 0.386 & 0.429 & 0.450 & 3.668 & 0.228 \\
\hline thiophene & 0.977 & 0.973 & 0.981 & 2.642 & 1.552 \\
\hline formaldehyde & 0.134 & 0.155 & 0.164 & 1.893 & -0.127 \\
\hline propionaldehyde & 0.539 & 0.533 & 0.522 & 2.315 & -0.205 \\
\hline butyraldehyde & 0.681 & 0.726 & 0.611 & 2.629 & 0.299 \\
\hline ethene & & & & 0.002 & 0.942 \\
\hline propene & & & & 0.402 & 1.372 \\
\hline 1-butene & & & & 0.673 & 1.683 \\
\hline 1,3-butadiene & & & & 0.939 & 1.389 \\
\hline carbon dioxide & & & & 0.259 & 0.339 \\
\hline
\end{tabular}


Table 3. Infinite Dilution Activity Coefficients and Logarithm of Partition Coefficients, $\log K_{\mathrm{L}}$ and $\log P$, of Organic Compounds in $\left[\mathrm{D}_{2} \mathrm{MIm}\right]^{+}\left[\mathrm{Tf}_{2} \mathrm{~N}\right]$

\begin{tabular}{|c|c|c|c|c|c|}
\hline \multirow{3}{*}{$\begin{array}{l}\text { solute } \\
\text { hexane }\end{array}$} & \multicolumn{3}{|c|}{$\gamma^{\infty}$} & \multirow{2}{*}{\multicolumn{2}{|c|}{$\frac{\log K_{\mathrm{L}} \quad \log P}{298.15 \mathrm{~K}}$}} \\
\hline & $323.10 \mathrm{~K}$ & $333.15 \mathrm{~K}$ & $343.15 \mathrm{~K}$ & & \\
\hline & 1.825 & 1.792 & 1.756 & 2.074 & 3.894 \\
\hline 3-methylpentane & 1.708 & 1.675 & 1.633 & 2.006 & 3.846 \\
\hline heptane & 2.531 & 2.111 & 2.077 & 2.261 & 4.221 \\
\hline octane & 2.538 & 2.484 & 2.448 & 2.947 & 5.057 \\
\hline nonane & 3.306 & 3.172 & 3.448 & 3.357 & 5.507 \\
\hline decane & 3.562 & 3.461 & 3.328 & 3.793 & 6.113 \\
\hline methylcyclopentane & 1.354 & 1.326 & 1.301 & 2.243 & 3.413 \\
\hline cyclohexane & 1.318 & 1.285 & 1.256 & 2.396 & 3.296 \\
\hline methylcyclohexane & 1.517 & 1.484 & 1.454 & 2.660 & 3.870 \\
\hline cycloheptane & 3.167 & 4.458 & 6.093 & 2.980 & 3.570 \\
\hline benzene & 0.429 & 0.431 & 0.434 & 2.930 & 2.300 \\
\hline toluene & 0.512 & 0.520 & 0.527 & 3.373 & 2.723 \\
\hline ethylbenzene & 0.652 & 0.662 & 0.689 & 3.723 & 3.143 \\
\hline$m$-xylene & 0.645 & 0.653 & 0.663 & 3.805 & 3.195 \\
\hline$p$-xylene & 0.647 & 0.660 & 0.657 & 3.794 & 3.204 \\
\hline$o$-xylene & 0.626 & 0.616 & 0.603 & 3.878 & 3.218 \\
\hline 1-hexene & 1.393 & 1.376 & 1.364 & 2.111 & 3.271 \\
\hline 1-hexyne & 0.925 & 0.917 & 0.899 & 2.456 & 2.666 \\
\hline 1-heptyne & 1.065 & 1.060 & 1.061 & 2.887 & 3.327 \\
\hline 2-butanone & 0.318 & 0.292 & 0.264 & 3.035 & 0.315 \\
\hline 2-pentanone & 0.397 & 0.404 & 0.407 & 3.409 & 0.829 \\
\hline 3-pentanone & 0.37 & 0.378 & 0.386 & 3.397 & 0.897 \\
\hline 1,4-dioxane & 0.463 & 0.460 & 0.453 & 3.309 & -0.401 \\
\hline methanol & 0.963 & 0.857 & 0.755 & 2.312 & -1.428 \\
\hline ethanol & 1.271 & 1.150 & 1.041 & 2.536 & -1.134 \\
\hline 1-propanol & 1.307 & 1.160 & 1.061 & 2.94 & -0.620 \\
\hline 2-propanol & 1.267 & 1.140 & 1.035 & 2.657 & -0.823 \\
\hline 2-methyl-1-propanol & 1.300 & 1.171 & 1.062 & 3.215 & -0.085 \\
\hline 1-butanol & 1.376 & 1.202 & 1.117 & 3.392 & -0.068 \\
\hline trifluoroethanol & 0.399 & 0.379 & 0.355 & 3.013 & -0.147 \\
\hline diethyl ether & 0.746 & 0.771 & 0.773 & 1.978 & 0.578 \\
\hline diisopropyl ether & 1.122 & 1.232 & 1.233 & 2.416 & 1.386 \\
\hline chloroform & 0.374 & 0.395 & 0.402 & 2.731 & 1.941 \\
\hline dichloromethane & 0.330 & 0.341 & 0.351 & 2.275 & 1.315 \\
\hline tetrachloromethane & 0.776 & 0.777 & 0.777 & 2.589 & 2.779 \\
\hline acetonitrile & 0.470 & 0.453 & 0.440 & 2.875 & 0.025 \\
\hline nitromethane & 0.596 & 0.559 & 0.536 & 3.125 & 0.175 \\
\hline 1-nitropropane & 0.504 & 0.487 & 0.479 & 3.777 & 1.327 \\
\hline triethylamine & 3.672 & 3.174 & 2.863 & 2.013 & -0.347 \\
\hline pyridine & 0.328 & 0.331 & 0.334 & 3.699 & 0.259 \\
\hline thiophene & 0.422 & 0.423 & 0.424 & 3.007 & 1.977 \\
\hline formaldehyde & 0.074 & 0.080 & 0.085 & 2.180 & 0.160 \\
\hline
\end{tabular}

similarly to 1-ethyl-3-methylimidazolium dicyanamide and appears to be a good choice for separating hexane/methanol, hexane/thiophene, and cyclohexane/thiophene mixtures. Results for the three other ILs studied suggest they will not attract particular interest for these specific separation problems.

Linear Solvation Energy Relationship (LSER) Characterization. In Table 1, there are 45 experimental $\log K_{\mathrm{L}}$ and $\log P$ values
Table 4. Infinite Dilution Activity Coefficients and Logarithm of Partition Coefficients, $\log K_{\mathrm{L}}$ and $\log P$, of Organic Compounds in $[\mathrm{EMIm}]^{+}\left[\mathrm{MeSO}_{3}\right]^{-}$

\begin{tabular}{|c|c|c|c|c|c|}
\hline \multirow{2}{*}{ solute } & \multicolumn{3}{|c|}{$\gamma^{\infty}$} & \multicolumn{2}{|c|}{$\log K_{\mathrm{L}} \quad \log P$} \\
\hline & $312.55 \mathrm{~K}$ & $322.45 \mathrm{~K}$ & $332.45 \mathrm{~K}$ & \multicolumn{2}{|c|}{$298.15 \mathrm{~K}$} \\
\hline hexane & 206.773 & 189.568 & 152.483 & 0.304 & 2.124 \\
\hline 3-methylpentane & 156.837 & 135.415 & 115.201 & 0.333 & 2.173 \\
\hline heptane & 432.487 & 2232.657 & 496.685 & 0.666 & 2.626 \\
\hline 2,2,4-trimethylpentane & 445.177 & 386.576 & 333.734 & 0.472 & 2.592 \\
\hline octane & 588.387 & 556.416 & 184.450 & 0.963 & 3.073 \\
\hline nonane & 1047.037 & 925.051 & 483.466 & 1.024 & 3.174 \\
\hline decane & 1408.052 & 1303.804 & 1158.638 & 1.558 & 3.818 \\
\hline undecane & 2011.800 & 1893.008 & 1717.795 & 1.926 & 4.306 \\
\hline dodecane & 2552.251 & 2422.466 & 2292.163 & 2.315 & 4.845 \\
\hline tridecane & 3376.144 & 3276.796 & 3068.670 & 2.712 & \\
\hline tetradecane & 4065.351 & 4003.456 & 3828.889 & 3.128 & \\
\hline methylcyclopentane & 102.064 & 95.800 & 79.455 & 0.673 & 1.843 \\
\hline cyclohexane & 96.795 & 88.264 & 76.345 & 0.853 & 1.753 \\
\hline methylcyclohexane & 169.213 & 131.109 & 130.385 & 0.946 & 2.196 \\
\hline cycloheptane & 194.190 & 250.689 & 322.731 & 1.335 & 1.915 \\
\hline benzene & 4.306 & 4.271 & 4.235 & 2.297 & 1.667 \\
\hline toluene & 13.109 & 8.735 & 8.569 & 2.187 & 1.537 \\
\hline ethylbenzene & 23.694 & 16.842 & 16.470 & 2.427 & 1.847 \\
\hline$m$-xylene & 19.280 & 18.753 & 18.224 & 2.688 & 2.078 \\
\hline$p$-xylene & 18.073 & 17.795 & 17.311 & 2.694 & 2.104 \\
\hline$o$-xylene & 14.725 & 14.514 & 14.256 & 2.907 & 2.247 \\
\hline 1-hexene & 86.953 & 83.446 & 68.874 & 0.628 & 1.788 \\
\hline 1-hexyne & 10.621 & 10.699 & 10.664 & 1.785 & 1.995 \\
\hline 1-heptyne & 19.195 & 19.396 & 19.386 & 2.020 & 2.460 \\
\hline 2-butanone & 3.930 & 3.686 & 3.348 & 2.336 & -0.384 \\
\hline 2-pentanone & 7.789 & 7.704 & 7.571 & 2.470 & -0.110 \\
\hline 3-pentanone & 7.576 & 7.451 & 7.371 & 2.439 & -0.061 \\
\hline 1,4-dioxane & 2.675 & 2.666 & 2.660 & 2.936 & -0.774 \\
\hline methanol & 0.249 & 0.254 & 0.264 & 3.439 & -0.301 \\
\hline ethanol & 0.438 & 0.418 & 0.405 & 3.476 & -0.194 \\
\hline 1-propanol & 0.742 & 0.734 & 0.722 & 3.704 & 0.144 \\
\hline 2-propanol & 0.924 & 0.907 & 0.877 & 3.290 & -0.190 \\
\hline diethyl ether & 21.818 & 20.373 & 18.006 & 0.779 & -0.391 \\
\hline diisopropyl ether & 84.519 & 74.151 & 67.694 & 0.739 & -0.291 \\
\hline chloroform & 0.563 & 0.479 & 1.418 & 2.762 & 1.972 \\
\hline dichloromethane & 0.471 & 0.531 & 1.299 & 2.530 & 1.570 \\
\hline tetrachloromethane & 4.572 & 4.793 & 0.669 & 2.243 & 2.433 \\
\hline acetonitrile & 1.263 & 0.818 & 0.836 & 2.709 & -0.141 \\
\hline nitromethane & 2.057 & 1.261 & 0.801 & 2.716 & -0.234 \\
\hline 1-nitropropane & 4.190 & 2.702 & 1.726 & 2.978 & 0.528 \\
\hline triethylamine & 8.322 & 14.329 & 18.845 & 2.441 & 0.081 \\
\hline thiophene & 1.871 & 1.905 & 1.951 & 2.755 & 1.665 \\
\hline formaldehyde & 0.615 & 0.656 & 0.691 & - & - \\
\hline propionaldehyde & 2.756 & 2.714 & 2.652 & 1.974 & -0.546 \\
\hline butyraldehyde & 4.869 & 4.947 & 3.509 & 2.056 & -0.274 \\
\hline
\end{tabular}

for solutes dissolved in $[\mathrm{HMIm}]^{+}[\mathrm{FAP}]^{-}$at $298 \mathrm{~K}$. Preliminary regression analyses on the entire data set indicated that dichloromethane was a statistical outlier. The compound was removed, and analysis of the remaining 44 tabulated experimental data 
points gave:

$$
\begin{aligned}
\log K_{\mathrm{L}}= & -0.324(0.111)-0.242(0.121) E+2.312(0.111) S \\
& +1.162(0.167) A+0.672(0.118) B+0.729(0.030) L \\
(N= & \left.44, \mathrm{SD}=0.124, R^{2}=0.969, F=237.2\right) \quad(9) \\
\log P= & -0.058(0.164)-0.138(0.149) E+0.616(0.150) S \\
& -2.420(0.208) A-4.287(0.146) B+3.554(0.130) V \\
(N= & \left.44, \mathrm{SD}=0.156, R^{2}=0.994, F=1174.5\right) \quad(10)
\end{aligned}
$$

Table 5. Activity Coefficients at Infinite Dilution of Various Organic Compounds in $[\mathrm{EMIm}]^{+}\left[\mathrm{MeSO}_{3}\right]^{-}$at $323.15 \mathrm{~K}$

\begin{tabular}{lccc} 
& Blahut et al. $^{39}$ & & this work \\
\cline { 2 - 3 } \multicolumn{1}{c}{ solute } & $T=323.15 \mathrm{~K}$ & & $T=322.45 \mathrm{~K}$ \\
methylcyclohexane & 198 & & 131 \\
$m$-xylene & 20.5 & 18.75 \\
benzene & 4.5 & 4.271 \\
methanol & 0.239 & 0.254 \\
chloroform & 0.515 & 0.479 \\
acetonitrile & 0.873 & 0.818 \\
thiophene & 2.06 & 1.905
\end{tabular}

where $N$ denotes the number of experimental values used in the regression analysis, $S D$ refers to the standard deviation, $R^{2}$ is the squared correlation coefficient, and $F$ is the Fisher F-statistic. All regression analyses were performed using SPSS statistical software. The standard errors in the calculated coefficients are given parenthetically immediately following the respective coefficient. Solute descriptors used in the analysis are tabulated in Table 7. Equations 9 and 10 prove to be a very accurate mathematical description of the $\log K_{\mathrm{L}}$ and $\log P$ values for the studied solutes dissolved in $[\mathrm{HMIm}]^{+}[\mathrm{FAP}]^{-}$and can be used to estimate $\log K_{\mathrm{L}}$ and $\log P$ values for additional solutes in this IL.

As noted above, each of the calculated equation coefficients corresponds to the sum of the respective cation- and anionspecific contributions. We have reported ${ }^{35} \log K_{\mathrm{L}}$ equation coefficients of $c_{\text {cation }}=-0.395, e_{\text {cation }}=-0.062, s_{\text {cation }}=1.975$, $a_{\text {cation }}=2.234, b_{\text {cation }}=0.621$, and $l_{\text {cation }}=0.768$ for the $[\mathrm{HMIm}]^{+}$cation. Equation coefficients of $c_{\text {anion }}=0.071, e_{\text {anion }}=$ $-0.180, s_{\text {anion }}=0.337, a_{\text {anion }}=-1.072, b_{\text {anion }}=0.051$, and $l_{\text {anion }}=$ -0.039 are calculated for the $[\mathrm{FAP}]^{-}$anion by subtracting the $[\mathrm{HMIm}]^{+}$equation coefficients from those in eq 9. Anion-

\begin{tabular}{|c|c|c|c|c|c|}
\hline \multicolumn{2}{|r|}{ ILs } & \multicolumn{4}{|c|}{$S_{12}^{\infty} / k_{1}^{\infty}$} \\
\hline anion & cation & $\begin{array}{l}\text { hexane/ } \\
\text { benzene }\end{array}$ & $\begin{array}{l}\text { hexane/ } \\
\text { methanol }\end{array}$ & $\begin{array}{l}\text { hexane/ } \\
\text { thiophene }\end{array}$ & $\begin{array}{l}\text { cyclohexane/ } \\
\text { thiophene }\end{array}$ \\
\hline \multirow{15}{*}[\mathrm{Tf}_{2}\mathrm{N}]{} & $\begin{array}{l}\text { 1-hexyl-3-methylimidazolium } \\
\text { tris(pentafluoroethyl)trifluorophosphate, } \\
{[\mathrm{HMIm}]^{+}[\mathrm{FAP}]^{-}}\end{array}$ & $12.38 / 1.93$ & $4.89 / 0.81$ & $6.16 / 1.02$ & $7.14 / 1.02$ \\
\hline & $\begin{array}{l}\text { 1-butyl-3-methylimidazolium } \\
\text { bis(pentafluoroethylsulfonyl)imide, } \\
{[\mathrm{BMIm}]^{+}[\mathrm{BETI}]^{-}}\end{array}$ & $9.37 / 0.99$ & $5.69 / 0.81$ & $9.68 / 1.02$ & $7.14 / 1.02$ \\
\hline & $\begin{array}{l}\text { 1,3-didecyl-2-methylimidazolium } \\
\text { bis(trifluoromethylsulfonyl)imide, } \\
{\left[\mathrm{D}_{2} \mathrm{MIm}\right]^{+}\left[\mathrm{Tf}_{2} \mathrm{~N}\right]^{-}}\end{array}$ & $4.23 / 2.32$ & $1.89 / 4$ & $4.33 / 2.38$ & $3.14 / 2.38$ \\
\hline & $\begin{array}{l}\text { 1-ethyl-3-methylimidazolium } \\
\text { methanesulfonate, }[\mathrm{EMIm}]^{+}\left[\mathrm{MeSO}_{3}\right]^{-}\end{array}$ & $48.1 / 0.23$ & $826 / 4$ & $110 / 0.53$ & $51.9 / 0.53$ \\
\hline & 1,3-dimethoxyimidazolium & $21.3 / 0.47$ & $42.05 / 0.94$ & $24.8 / 0.94$ & $12.6 / 0.94$ \\
\hline & 1-(methylethylether)-3-methylimidazolium & $15.5 / 0.85$ & $17.4 / 0.93$ & $18.1 / 1.0$ & 10.9 \\
\hline & 1-ethanol-3-methylimidazolium & $20.6 / 0.47$ & $49.1 / 1.12$ & $24.7 / 0.56$ & $14.2 / 0.56$ \\
\hline & 1-ethyl-3-methylimidazolium & $37.5 / 1.43$ & $19.5 / 1.20$ & - & - \\
\hline & 1-(hexylmethylether)-3-methylimidazolium & $9.1 / 1.23$ & $6.8 / 0.91$ & $10.0 / 1.35$ & $6.4 / 1.35$ \\
\hline & 1,3-bis(hexylmethylether)imidazolium & $4.9 / 1.67$ & $3.2 / 1.06$ & $5.3 / 1.75$ & $3.7 / 1.75$ \\
\hline & 1-butyl-3-methylimidazolium & $16.7 / 1.11$ & - & - & - \\
\hline & 1-hexyl-3-methylimidazolium & $9.5 / 1.29$ & $6.1 / 0.82$ & - & - \\
\hline & trimethylhexylammonium & $9.9 / 1.01$ & $8.5 / 0.86$ & $10.7 / 1.09$ & $7.2 / 1.09$ \\
\hline & 4-methyl- $N$-butyl-pyridinium & $18.8 / 1.43$ & $21.2 / 0.83$ & $10.6 / 1.56$ & $6.1 / 1.56$ \\
\hline & triethylsulphonium & 21.6/0.91 & $17.8 / 0.77$ & $25.5 / 1.05$ & $14.3 / 1.05$ \\
\hline \multirow{3}{*}[\mathrm{DCA}]{} & trihexyl(tetradecyl) phosphonium & $2.7 / 2.56$ & $1.1 / 1.02$ & $2.6 / 2.5$ & $1.95 / 2.5$ \\
\hline & 1-cyanopropyl-3-methylimidaolium & $56.0 / 0.22$ & $432 / 1.69$ & $105 / 0.41$ & $41.3 / 0.41$ \\
\hline & 1-ethyl-3-methylimidazolium & $43.4 / 0.39$ & $255 / 2.27$ & $69.6 / 0.63$ & $28.8 / 0.63$ \\
\hline$\left[\mathrm{BF}_{4}\right]$ & 1-ethanol-3-methylimidazolium & $-/ 0.10$ & $-/ 0.98$ & $-/ 0.17$ & $136.1 / 0.17$ \\
\hline$\left[\mathrm{PF}_{6}\right]$ & 1-ethanol-3-methylimidazolium & $-/ 0.17$ & $-/ 0.77$ & $-/ 0.23$ & $59.7 / 0.23$ \\
\hline
\end{tabular}
specific equation coefficients of $c_{\text {anion }}=-0.005, e_{\text {anion }}=$ $-0.249, s_{\text {anion }}=0.383, a_{\text {anion }}=-1.062, b_{\text {anion }}=0.084$, and $v_{\text {anion }}=$ -0.051 for $[\mathrm{FAP}]^{-}$for the $\log P$ correlation were calculated in a similar fashion. The calculated anion-specific equation

\section{Table 6. Selectivities $S_{12}^{\infty}$ and Capacities $k_{1}^{\infty}$ at Infinite Dilution for Different Separation Problems at 323.15 K}


Table 7. Solute Descriptors of Compounds Used in the Study

\begin{tabular}{|c|c|c|c|c|c|c|}
\hline solute & $E$ & $S$ & $A$ & $B$ & $L$ & $V$ \\
\hline hexane & 0.000 & 0.000 & 0.000 & 0.000 & 2.668 & 0.9540 \\
\hline 3-methylpentane & 0.000 & 0.000 & 0.000 & 0.000 & 2.581 & 0.9540 \\
\hline heptane & 0.000 & 0.000 & 0.000 & 0.000 & 3.173 & 1.0949 \\
\hline 2,2,4-trimethylpentane & 0.000 & 0.000 & 0.000 & 0.000 & 3.106 & 1.2358 \\
\hline octane & 0.000 & 0.000 & 0.000 & 0.000 & 3.677 & 1.2358 \\
\hline nonane & 0.000 & 0.000 & 0.000 & 0.000 & 4.182 & 1.3767 \\
\hline decane & 0.000 & 0.000 & 0.000 & 0.000 & 4.686 & 1.5176 \\
\hline undecane & 0.000 & 0.000 & 0.000 & 0.000 & 5.191 & 1.6590 \\
\hline dodecane & 0.000 & 0.000 & 0.000 & 0.000 & 5.696 & 1.7994 \\
\hline tridecane & 0.000 & 0.000 & 0.000 & 0.000 & 6.200 & 1.9400 \\
\hline tetradecane & 0.000 & 0.000 & 0.000 & 0.000 & 6.705 & 2.0810 \\
\hline methylcyclopentane & 0.225 & 0.100 & 0.000 & 0.000 & 2.907 & 0.8454 \\
\hline cyclohexane & 0.310 & 0.100 & 0.000 & 0.000 & 2.964 & 0.8454 \\
\hline methylcyclohexane & 0.244 & 0.060 & 0.000 & 0.000 & 3.319 & 0.9863 \\
\hline cycloheptane & 0.350 & 0.100 & 0.000 & 0.000 & 3.704 & 0.9863 \\
\hline benzene & 0.610 & 0.520 & 0.000 & 0.140 & 2.786 & 0.7164 \\
\hline toluene & 0.601 & 0.520 & 0.000 & 0.140 & 3.325 & 0.8573 \\
\hline ethylbenzene & 0.613 & 0.510 & 0.000 & 0.150 & 3.778 & 0.9982 \\
\hline$m$-xylene & 0.623 & 0.520 & 0.000 & 0.160 & 3.839 & 0.9982 \\
\hline$p$-xylene & 0.613 & 0.520 & 0.000 & 0.160 & 3.839 & 0.9982 \\
\hline$o$-xylene & 0.663 & 0.560 & 0.000 & 0.160 & 3.939 & 0.9982 \\
\hline 1-hexene & 0.080 & 0.080 & 0.000 & 0.070 & 2.572 & 0.9110 \\
\hline 1-hexyne & 0.166 & 0.220 & 0.100 & 0.120 & 2.510 & 0.8680 \\
\hline 1-heptyne & 0.160 & 0.230 & 0.090 & 0.100 & 3.000 & 1.0089 \\
\hline 2-butanone & 0.166 & 0.700 & 0.000 & 0.510 & 2.287 & 0.6879 \\
\hline 2-pentanone & 0.143 & 0.680 & 0.000 & 0.510 & 2.755 & 0.8288 \\
\hline 3-pentanone & 0.154 & 0.660 & 0.000 & 0.510 & 2.811 & 0.8288 \\
\hline 1,4 dioxane & 0.329 & 0.750 & 0.000 & 0.640 & 2.892 & 0.6810 \\
\hline methanol & 0.278 & 0.440 & 0.430 & 0.470 & 0.970 & 0.3082 \\
\hline ethanol & 0.246 & 0.420 & 0.370 & 0.480 & 1.485 & 0.4491 \\
\hline 1-propanol & 0.236 & 0.420 & 0.370 & 0.480 & 2.031 & 0.5900 \\
\hline 2-propanol & 0.212 & 0.360 & 0.330 & 0.560 & 1.764 & 0.5900 \\
\hline 2-methyl-1-propanol & 0.217 & 0.390 & 0.370 & 0.480 & 2.413 & 0.7309 \\
\hline 1-butanol & 0.224 & 0.420 & 0.370 & 0.480 & 2.601 & 0.7309 \\
\hline trifluoroethanol & 0.015 & 0.600 & 0.570 & 0.250 & 1.224 & 0.5022 \\
\hline diethyl ether & 0.041 & 0.250 & 0.000 & 0.450 & 2.015 & 0.7309 \\
\hline diisopropyl ether & -0.063 & 0.170 & 0.000 & 0.570 & 2.501 & 1.0127 \\
\hline chloroform & 0.425 & 0.490 & 0.150 & 0.020 & 2.480 & 0.6167 \\
\hline dichloromethane & 0.390 & 0.570 & 0.100 & 0.050 & 2.019 & 0.4943 \\
\hline tetrachloromethane & 0.460 & 0.380 & 0.000 & 0.000 & 2.823 & 0.7391 \\
\hline acetonitrile & 0.237 & 0.900 & 0.070 & 0.320 & 1.739 & 0.4042 \\
\hline nitromethane & 0.313 & 0.950 & 0.060 & 0.310 & 1.892 & 0.4237 \\
\hline 1-nitropropane & 0.242 & 0.950 & 0.000 & 0.310 & 2.894 & 0.7055 \\
\hline triethylamine & 0.101 & 0.150 & 0.000 & 0.790 & 3.040 & 1.0538 \\
\hline pyridine & 0.631 & 0.840 & 0.000 & 0.520 & 3.022 & 0.6753 \\
\hline thiophene & 0.687 & 0.570 & 0.000 & 0.150 & 2.819 & 0.6411 \\
\hline propionaldehyde & 0.196 & 0.650 & 0.000 & 0.450 & 1.815 & 0.5470 \\
\hline butyraldehyde & 0.187 & 0.650 & 0.000 & 0.450 & 2.270 & 0.6879 \\
\hline ethene & 0.107 & 0.100 & 0.000 & 0.070 & 0.289 & 0.3470 \\
\hline propene & 0.100 & 0.080 & 0.000 & 0.070 & 0.946 & 0.4880 \\
\hline 1-butene & 0.100 & 0.080 & 0.000 & 0.070 & 1.529 & 0.6290 \\
\hline 1,3-butadiene & 0.320 & 0.230 & 0.000 & 0.100 & 1.543 & 0.5862 \\
\hline carbon dioxide & 0.000 & 0.280 & 0.050 & 0.100 & 0.058 & 0.2810 \\
\hline
\end{tabular}

coefficients that have been calculated for the $[\mathrm{FAP}]^{-}$can be combined with our previously reported cation-specific equation coefficients ${ }^{35}$ to enable $\log K_{\mathrm{L}}$ and $\log P$ predictions for additional ILs containing the $[\mathrm{FAP}]^{-}$anion.

The experimental $\log K_{\mathrm{L}}$ and $\log P$ data compiled in Table 2 for solutes dissolved in $[\mathrm{BMIm}]^{+}[\mathrm{BETI}]^{-}$at $298 \mathrm{~K}$ can be used to calculate the anion-specific equation coefficients for the $[\mathrm{BETI}]^{-}$anion. Included as the last five entries in Table 2 are $\log K_{\mathrm{L}}$ and $\log P$ values determined for gaseous solutes, including four alkenes (ethene, propene, 1-butene, 1,3-butadiene) and carbon dioxide, all of which were calculated from published Henry's law constants reported earlier by Kilaru and Scovazzo. ${ }^{40}$ The analysis of the $\log K_{\mathrm{L}}$ and $\log P$ data in Table 2 yields the following two Abraham model correlations:

$$
\begin{aligned}
\log K_{\mathrm{L}}= & -0.460(0.046)+0.141(0.084) E+2.206(0.076) S \\
& +1.980(0.108) A+0.696(0.083) B+0.613(0.012) L \\
(N= & \left.53, \mathrm{SD}=0.093, R^{2}=0.989, F=860.1\right)
\end{aligned}
$$

$$
\begin{aligned}
\log P= & 0.023(0.072)+0.083(0.099) E+0.334(0.095) S \\
& -1.701(0.130) A-4.236(0.098) B+3.041(0.062) V \\
(N & \left.=51, \mathrm{SD}=0.110, R^{2}=0.996, F=2094\right)
\end{aligned}
$$

The derived correlation models describe the observed $\log K_{\mathrm{L}}$ and $\log P$ results to within standard deviations of $\mathrm{SD}=0.093$ (eq 11) and $\mathrm{SD}=0.110$ (eq 12) log units, respectively. The calculated anion-specific equation coefficients for the $[\mathrm{BETI}]^{-}$ anion for the $\log K_{\mathrm{L}}$ correlation model are: $c_{\text {anion }}=-0.033, e_{\text {anion }}$ $=0.004, s_{\text {anion }}=0.245, a_{\text {anion }}=0.199, b_{\text {anion }}=0.250$, and $l_{\text {anion }}=$ 0.081 . These values were computed by subtracting the published cation-specific equation coefficient values ${ }^{35}$ of $c_{\text {cation }}=-0.427$, $e_{\text {cation }}=0.137, s_{\text {cation }}=1.961, a_{\text {cation }}=2.179, b_{\text {cation }}=0.946$, and $l_{\text {cation }}=0.694$ for $[\mathrm{BMIm}]^{+}$from the coefficients in eq 11 . The $\log P$ anion-specific equation coefficients for $[\mathrm{BETI}]^{-}$of $c_{\text {anion }}=$ $0.068, e_{\text {anion }}=-0.334, s_{\text {anion }}=0.217, a_{\text {anion }}=-0.196, b_{\text {anion }}=$ -0.238 , and $v_{\text {anion }}=-0.279$ were computed in similar fashion.

Assembled in Table 3 are the partition coefficient data for solutes dissolved in $\left[\mathrm{D}_{2} \mathrm{MIm}\right]^{+}\left[\mathrm{Tf}_{2} \mathrm{~N}\right]^{-}$at $298 \mathrm{~K}$. The analysis of the tabulated $\log K_{\mathrm{L}}$ and $\log P$ values yields the following expressions:

$$
\begin{aligned}
\log K_{\mathrm{L}}= & -0.252(0.089)-0.269(0.088) E+1.603(0.082) S \\
& +1.946(0.125) A+0.354(0.093) B+0.856(0.027) L \\
(N= & \left.40, \mathrm{SD}=0.082, R^{2}=0.979, F=315.3\right)
\end{aligned}
$$

$$
\begin{aligned}
& \log P=-0.093(0.147)-0.052(0.118) E+0.040(0.128) S \\
&-1.620(0.176) A-4.667(0.132) B+4.034(0.132) V \\
&\left(N=40, \mathrm{SD}=0.118, R^{2}=0.996, F=1816\right)
\end{aligned}
$$

Triethylamine was removed from the final regression analysis as its calculated $\log K_{\mathrm{L}}$ and $\log P$ values differ from the observed values by more than three standard deviations. Equations 13 and 14 describe the observed $\log K_{\mathrm{L}}$ and $\log P$ data to within standard deviations of 0.082 and $0.118 \mathrm{log}$ units for the remaining 40 solutes. The calculated coefficients in eqs 13 and 14 correspond to the cation-specific values for the $\left[\mathrm{D}_{2} \mathrm{MIm}\right]^{+}$cation itself. This is true because in establishing our computation methodology, the equation coefficients for the $\left[\mathrm{Tf}_{2} \mathrm{~N}\right]^{-}$anion were set equal to 
zero to provide a reference point from which all other equation coefficients would be calculated.

In Table 4, there are 44 experimental $\log K_{\mathrm{L}}$ and 42 experimental $\log P$ values for solutes dissolved in $[\mathrm{EMIm}]^{+}\left[\mathrm{MeSO}_{3}\right]^{-}$. Preliminary regression analyses on the entire data set indicated that triethylamine and carbon tetrachloride were statistical outliers. These two solutes were eliminated from the data set, and the regression analysis performed to yield

$$
\begin{aligned}
\log K_{\mathrm{L}}= & -1.398(0.119)+0.485(0.162) E+2.562(0.160) S \\
& +6.616(0.275) A+0.495(0.198) B+0.642(0.028) L \\
(N= & \left.42, \mathrm{SD}=0.153, R^{2}=0.976, F=288.5\right)
\end{aligned}
$$

$$
\begin{aligned}
\log P= & -0.799(0.207)+0.493(0.204) E+0.644(0.217) S \\
& +2.842(0.363) A-4.440(0.243) B+3.007(0.164) V \\
(N= & \left.40, \mathrm{SD}=0.189, R^{2}=0.981, F=393.6\right)
\end{aligned}
$$

Equations 15 and 16 describe experimental $\log K_{\mathrm{L}}$ and $\log P$ data to within standard deviations of $\mathrm{SD}=0.153$ and $\mathrm{SD}=0.189$ $\log$ units, respectively. As was the case for all four ILs studied, a slightly larger deviation was noted for the $\log P$ correlation. The slightly larger standard deviation is to be expected as the $\log P$ values also contain the experimental uncertainty associated with $\log K_{\mathrm{w}}$ data that were used to convert the measured $\log K_{\mathrm{L}}$ data to $\log P$ via eq 6 . The calculated anion-specific equation coefficients for $\left[\mathrm{MeSO}_{3}\right]^{-}$based on the $\log K_{\mathrm{L}}$ correlation are: $c_{\text {anion }}=$ $-0.872, e_{\text {anion }}=0.234, s_{\text {anion }}=0.276, a_{\text {anion }}=-1.703, b_{\text {anion }}=$ -0.552 , and $l_{\text {anion }}=0.001$. These values were computed by subtracting the published cation-specific equation coefficient values $^{35}$ of $c_{\text {cation }}=-0.526, e_{\text {cation }}=0.248, s_{\text {cation }}=2.286, a_{\text {cation }}=$ $2.219, b_{\text {cation }}=1.047$, and $l_{\text {cation }}=0.641$ for $[E M I m]^{+}$from the coefficients in eq 15 . The $\log P$ anion-specific equation coefficients for $\left[\mathrm{MESO}_{3}\right]^{-}$of $c_{\text {anion }}=0.824, e_{\text {anion }}=0.094, s_{\text {anion }}=$ $0.400, a_{\text {anion }}=4.289, b_{\text {anion }}=-0.742$, and $v_{\text {anion }}=-0.098$ were computed likewise.

\section{CONCLUDING REMARKS}

Partition and infinite dilution activity coefficients of organic compounds in the four new ILs $[\mathrm{HMIm}]^{+}[\mathrm{FAP}]^{-},[\mathrm{BMIm}]^{+}$$[\mathrm{BETI}]^{-},\left[\mathrm{D}_{2} \mathrm{MIm}\right]^{+}[\mathrm{Tf} 2 \mathrm{~N}]^{-}$, and $[\mathrm{EMIm}]^{+}\left[\mathrm{MeSO}_{3}\right]^{-}$were measured using inverse gas chromatography from (312 to 353 ) $\mathrm{K}$. The partition coefficients were also converted into water-to-IL partition coefficients using the corresponding gas-to-water partition coefficients. Both sets of partition coefficients were analyzed using the Abraham solvation parameter model with cationspecific and anion-specific equation coefficients. The derived equations correlated the experimental gas-to-IL and water-to-IL partition coefficient data to within average standard deviations of 0.113 and $0.143 \log$ units, respectively.

\section{AUTHOR INFORMATION}

\section{Corresponding Author}

*E-mail: fabrice.mutelet@ensic.inpl-nancy.fr. Telephone number: +33 3831751 31. Fax number: +33383175395.

\section{REFERENCES}

(1) Meindersma, G. W.; Galán Sánchez, L. M.; Hansmeier, A. R.; De Haan, A. B. Application of task-specific ionic liquids for intensified separations. Monatsh. Chem. 2007, 138, 1125-1136.
(2) Brennecke, J. F.; Maginn, E. J. Ionic liquids: Innovative fluids for chemical processing. AIChE J. 2001, 47, 2384-2389.

(3) Earle, M. J.; Seddon, K. R. Ionic liquids. Green solvents for the future. Pure Appl. Chem. 2000, 72, 1391-1398.

(4) Revelli, A. L.; Sprunger, L. M.; Gibbs, J.; Acree, W. E., Jr.; Baker, G. A.; Mutelet, F. Activity Coefficients at Infinite Dilution of Organic Compounds in Trihexyl(tetradecyl)phosphonium Bis(trifluoromethylsulfonyl)imide Using Inverse Gas Chromatography. J. Chem. Eng. Data 2009, 54, 977-985.

(5) Revelli, A. L.; Mutelet, F.; Turmine, M.; Solimando, R.; Jaubert, J. N. Activity Coefficients at Infinite Dilution of Organic Compounds in 1-Butyl-3-methylimidazolium Tetrafluoroborate Using Inverse Gas Chromatography. J. Chem. Eng. Data 2008, 54, 90-101.

(6) Mutelet, F.; Butet, V.; Jaubert, J. N. Application of Inverse Gas Chromatography and Regular Solution Theory for Characterization of Ionic Liquids. Ind. Eng. Chem. Res. 2005, 44, 4120-4127.

(7) Mutelet, F.; Jaubert, J. N. Accurate measurements of thermodynamic properties of solutes in ionic liquids using inverse gas chromatography. J. Chromatogr., A 2006, 1102, 256-267.

(8) Mutelet, F.; Jaubert, J. N.; Rogalski, M.; Boukherissa, M.; Dicko, A. Thermodynamic Properties of Mixtures Containing Ionic Liquids: Activity Coefficients at Infinite Dilution of Organic Compounds in 1-Propyl Boronic Acid-3-Alkylimidazolium Bromide and 1-Propenyl-3alkylimidazolium Bromide Using Inverse Gas Chromatography. J. Chem. Eng. Data 2006, 51, 1274-1279.

(9) Mutelet, F.; Jaubert, J. N.; Rogalski, M.; Harmand, J.; Sindt, M.; Mieloszynski, J. L. Activity Coefficients at Infinite Dilution of Organic Compounds in 1-Meth)acryloyloxyalkyl-3-methylimidazolium Bromide Using Inverse Gas Chromatography. J. Phys. Chem. B 2008, 112, 3773-3785.

(10) Mutelet, F.; Revelli, A. L.; Jaubert, J. N.; Sprunger, L. M.; Acree, W. E., Jr.; Baker, G. A. Partition Coefficients of Organic Compounds in New Imidazolium and Tetralkylammonium Based Ionic Liquids Using Inverse Gas Chromatography. J. Chem. Eng. Data 2010, 55, 234-242.

(11) Arlt, M.; Seiler, M.; Jork, C.; Schneider, T. DE Patent No. 10114734, 2001.

(12) Alonso, L.; Arce, A.; Francisco, M.; Soto, A. Solvent extraction of thiophene from $n$-alkanes $\left(\mathrm{C}_{7}, \mathrm{C}_{12}\right.$, and $\left.\mathrm{C}_{16}\right)$ using the ionic liquid $\left[\mathrm{C}_{8} \mathrm{mim}\right]\left[\mathrm{BF}_{4}\right]$. J. Chem. Thermodyn. 2008, 40, 966-972.

(13) Poole, C. F. Chromatographic and spectroscopic methods for the determination of solvent properties of room temperature ionic liquids. J. Chromatogr., A 2004, 1037, 49-82.

(14) Heintz, A. Recent developments in thermodynamics and thermophysics of non-aqueous mixtures containing ionic liquids. a review. J. Chem. Thermodyn. 2005, 37, 525-535.

(15) Deenadayalu, N.; Letcher, T. M.; Reddy, P. Determination of Activity Coefficients at Infinite Dilution of Polar and Nonpolar Solutes in the Ionic Liquid 1-Ethyl-3-methylimidazolium Bis(trifluoromethylsulfonyl) Imidate Using Gas-Liquid Chromatography at the Temperature 303.15 or 318.15 K. J. Chem. Eng. Data 2005, 50, 105-108.

(16) Krummen, M.; Wasserscheid, P.; Gmehling, J. Measurement of Activity Coefficients at Infinite Dilution in Ionic Liquids Using the Dilutor Technique. J. Chem. Eng. Data 2002, 7, 1411-1417.

(17) Letcher, T. M.; Soko, B.; Ramjugernath, D.; Deenadayalu, N.; Nevines, A.; Naicker, P. K. Activity Coefficients at Infinite Dilution of Organic Solutes in 1- Hexyl-3-Methylimidazolium Hexafluorophosphate from Gas-Liquid Chromatography. J. Chem. Eng. Data 2003, $48,708-711$.

(18) Domańska, U.; Marciniak, A. Physicochemical properties and activity coefficients at infinite dilution for organic solutes and water in the ionic liquid 1-decyl-3-methylimidazolium tetracyanoborate. J. Phys. Chem. B 2010, 114, 16542-16547.

(19) Domańska, U.; Marciniak, A.; Królikowska, M.; Arasimowicz, M. Activity coefficients at infinite dilution measurements for organic solutes and water in the ionic liquid 1-hexyl-3-methylimidazolium thiocyanate. J. Chem. Eng. Data 2010, 55, 2532-2536.

(20) Marciniak, A. Influence of cation and anion structure of the ionic liquid on extraction processes based on activity coefficients at infinite dilution. A review. Fluid Phase Equilib. 2010, 294, 213-233. 
(21) Freemantle, M. Ionic liquids in organic synthesis. Chem. Eng. News 2004, 82, 44-49.

(22) Zein El Abedin, S.; Borissenko, N.; Endres, F. Electropolymerization of benzene in a room temperature ionic liquid. Electrochem. Commun. 2004, 6, 422-426.

(23) Muldoon, M. J.; Aki, S. N. V. K.; Anderson, J. L.; Dixon, J. K.; Brennecke, J. F. Improving carbon dioxide solubility in ionic liquids. J. Phys. Chem. B 2007, 111, 9001-9009.

(24) Endres, F.; MacFarlane, D.; Abbott, A. Electropolymerization of benzene in a room temperature ionic liquid. Electrodeposition from Ionic Liquids; Wiley-VCH: New York, 2008.

(25) Chrobok, A.; Swadzba, M.; Baj, S. Oxygen solubility in ionic liquids based on 1-alkyl-3-methylimidazolium cations. Pol. J. Chem. 2007, 81, 337-344.

(26) Dyson, P. J.; Laurenczy, G.; Ohlin, A.; Vallance, J.; Welton, T. Determination of hydrogen concentration in ionic liquids and the effect (or lack of) on rates of hydrogenation. Chem. Commun. 2003, 2418-2419.

(27) O'Mahony, A. M.; Silvester, D. S.; Aldous, L.; Hardacre, C.; Compton, R. G. Effect of water on the electrochemical window and potential limits of room-temperature ionic liquids. J. Chem. Eng. Data 2008, 53, 2884-2891.

(28) Baker, S. N.; Baker, G. A. A Simple Colorimetric Assay of Ionic Liquid Hydrolytic Stability. Aust. J. Chem. 2005, 58, 174-177.

(29) Revelli, A.-L.; Mutelet, F.; Jaubert, J.-N.; Garcia-Martinez, M.; Sprunger, L. M.; Acree, W. E., Jr.; Baker, G. A. Study of ether-, alcohol-, or cyano-functionalized ionic liquids using inverse gas chromatography. J. Chem. Eng. Data 2010, 55, 2434-2443.

(30) Acree, W. E., Jr.; Abraham, M. H. The analysis of solvation in ionic liquids and organic solvents using the Abraham model linear free energy relationship. J. Chem. Technol. Biotechnol. 2006, 81, 1441-1446.

(31) Abraham, M. H.; Acree, W. E., Jr. Comparative analysis of solvation and selectivity in room temperature ionic liquids using the Abraham linear free energy relationship. Green Chem. 2006, 8, 906-915.

(32) Abraham, M. H.; Zissimos, A. M.; Huddleston, J. G.; Willauer, H. D.; Rogers, R. D.; Acree, W. E., Jr. Some novel liquid partitioning systems: Water-ionic liquids and aqueous biphasic systems. Ind. Eng. Chem. Res. 2003, 42, 413-418.

(33) Sprunger, L.; Clark, M.; Acree, W. E., Jr.; Abraham, M. H. Characterization of room-temperature ionic liquids by the Abraham model with cation-specific and anion-specific equation coefficients. J. Chem. Inf. Model. 2007, 47, 1123-1129.

(34) Sprunger, L. M.; Proctor, A.; Acree, W. E., Jr.; Abraham, M. H. LFER correlations for room temperature ionic liquids: Separation of equation coefficients into individual cation-specific and anion-specific contributions. Fluid Phase Equilib. 2008, 265, 104-111.

(35) Sprunger, L. M.; Gibbs, J.; Proctor, A.; Acree, W. E., Jr.; Abraham, M. H.; Meng, Y.; Yao, C.; Anderson, J. L. Linear Free Energy Relationship Correlations for Room Temperature Ionic Liquids: Revised Cation-Specific and Anion-Specific Equation Coefficients for Predictive Applications Covering a Much Larger Area of Chemical Space. Ind. Eng. Chem. Res. 2009, 48, 4145-4154.

(36) Burrell, A. K.; Del Sesto, R. E.; Baker, S. N.; McCleskey, T. M.; Baker, G. A. The Large Scale Synthesis of Pure Imidazolium and Pyrrolidinium Ionic Liquids. Green Chem. 2007, 9, 449-454.

(37) Baker, S. N.; McCleskey, T. M.; Pandey, S.; Baker, G. A. Fluorescence studies of protein thermostability in ionic liquids. Chem. Commun. 2004, 10, 940-941.

(38) Cruikshank, A. J. B.; Windsor, M. L.; Young, C. L. The use of gas chromatography to determine activity coefficients and second virial coefficients of mixtures. Proc. R. Soc. London 1966, A295, 259-270.

(39) Blahut, A.; Sobota, M.; Dohnal, V.; Vrbka, P. Activity coefficients at infinite dilution of organic solutes in the ionic liquid 1-ethyl-3methylimidazolium methanesulfonate. Fluid Phase Equilib. 2010, 299, 198-206.

(40) Kilaru, P. K.; Scovazzo, P. Correlations of low-pressure carbon dioxide and hydrocarbon solubilities in imidazolium-, phosphonium-, and ammonium-based room temperature ionic liquids. Part 2. Using activation energy of viscosity. Ind. Eng. Chem. Res. 2008, 47, 910-919. 